\title{
Poisson et pêche dans la littérature irlandaise et galloise: le bétail de la mer
}

Author(s): Natalia I. Petrovskaia

Source: Anthropozoologica, 53(1):139-146.

Published By: Muséum national d'Histoire naturelle, Paris

https://doi.org/10.5252/anthropozoologica2018v53a12

URL: http://www.bioone.org/doi/full/10.5252/anthropozoologica2018v53a12

BioOne (www.bioone.org) is a nonprofit, online aggregation of core research in the biological, ecological, and environmental sciences. BioOne provides a sustainable online platform for over 170 journals and books published by nonprofit societies, associations, museums, institutions, and presses.

Your use of this PDF, the BioOne Web site, and all posted and associated content indicates your acceptance of BioOne's Terms of Use, available at www.bioone.org/page/terms_of_use.

Usage of BioOne content is strictly limited to personal, educational, and non-commercial use. Commercial inquiries or rights and permissions requests should be directed to the individual publisher as copyright holder. 


\section{Poisson et pêche dans la littérature irlandaise et galloise: le bétail de la mer}

Natalia I. PETROVSKAIA

Universiteit Utrecht,

Trans 10, NL-3512 JK Utrecht (Pays-Bas)

n.petrovskaia@uu.nl

Soumis le 19 août 2017 | Accepté le 6 février 2018 | Publié le 24 août 2018

MOTS CLÉS

Littérature celtique, saumon, pêche,

monstres marins, représentations fictives de poissons.

KEY WORDS

Celtic Literature,

salmon,
medieval fishing,

marine monsters,

fictional representations

of fish.
Petrovskaia N. I. 2018. - Poisson et pêche dans la littérature irlandaise et galloise: le bétail de la mer, in Jacquemard C., Gauvin B., Lucas-Avenel M.-A., Clavel B. \& Buquet T. (éds), Animaux aquatiques et monstres des mers septentrionales (imaginer, connaître, exploiter, de l'Antiquité à 1600). Anthropozoologica 53 (12): 139-146. https://doi.org/10.5252/ anthropozoologica2018v53a12. http://anthropozoologica.com/53/12

\section{RÉSUMÉ}

Cet article traite de l'existence dans la littérature irlandaise, et dans une moindre mesure dans la littérature du Pays de Galles, de deux développements parallèles dans l'utilisation des références aux poissons et aux animaux aquatiques et marins. Le premier est l'utilisation de la symbolique, souvent analysée comme le résultat d'une évolution d'un récit mythologique, comme dans la légende galloise de Taliesin. Le deuxième, bien moins étudié, est l'utilisation descriptive liée à une expérience directe, ou aux attitudes sociales et culturelles, face à la pêche dans ces pays au Moyen Âge, comme par exemple dans la poésie irlandaise de la collection Agallamh na Senórach, «La conversation des anciens». Le but de cet article est de montrer que la symbolique (ou le mythologique) n'est qu'un aspect de la représentation de la nature dans la littérature irlandaise et la littérature galloise. Il sera montré, par une comparaison entre la Navigatio sancti Brendani, un texte latin de composition irlandaise, et le Voyage de Saint Brendan, son adaptation anglo-normande, que l'on observe une augmentation significative des éléments réalistes de la description des poissons et des êtres marins dans les textes dont le lectorat avait une connaissance directe de la mer et de la pêche.

\section{ABSTRACT}

Fish and fishing in medieval Irish and Welsh literature: the cattle of the sea.

This article deals with the existence in medieval Irish literature, and to a lesser extent in medieval Welsh literature, of two parallel developments in the use of references to fish and aquatic or marine animals. The first is the symbolic use, often analysed as the result of the evolution of mythological accounts, such as in the legend of Taliesin. The second, lesser studied, is the descriptive use, which represents direct experience, or perhaps even to some degree social and cultural attitudes to fishing in these countries in the Middle Ages, as in the Irish poetry of the Agallamb na Senórach "Colloquy of the ancients" collection, for instance. The purpose of this article is to show that the symbolic or the mythological is but one aspect of the representation of nature in medieval Irish and Welsh literatures. It will be shown by means of a comparison between the Navigatio sancti Brendani, a Latin text of Irish origins, and the Voyage de Saint Brendan, its Anglo-Norman adaptation, that the presence of realistic elements in the representation of marine life in a text is proportional to the direct experience of the sea and of fishing on the part of the text's audience. 


\section{INTRODUCTION}

Fégaid úaib sair fothúaid in muir múaid milach: adba rón rebach rán rogab lán linad. (Meyer 1919: 65). (Regardez devant vous vers le nord-ouest la mer puissante, riche en animaux:

le domicile des phoques agiles et magnifiques est la marée haute).

La citation ci-dessus est un court poème irlandais du IXe siècle, qui nous offre un exemple typique de la représentation de la nature marine dans la poésie irlandaise de cette période. Pourtant, le réalisme attesté par ce poème représente un aspect moins connu de la littérature irlandaise, car les études savantes des XXe et XXIe siècles ont souvent eu tendance à se concentrer sur les aspects fantastiques dans les littératures celtiques du Moyen Âge (l'irlandaise et la galloise en particulier). Le résultat de cette propension est que la représentation de la nature et du monde animal, notamment aquatique, dans les littératures irlandaise et galloise, est souvent perçue comme fantastique et étroitement liée à la mythologie. Comme l'observe Philippe Walter, qui analyse les expressions mythologiques liées aux mondes aquatiques dans la littérature des celtes médiévaux, les «peuples celtiques insulaires [...] ont toujours entretenu une relation privilégiée avec le milieu maritime" (Walter 2002: 237). Cette réflexion nous incite à étudier les différentes façons dont ce contact étroit avec la mer a influencé les littératures celtiques. Le but de cet article est de montrer que la symbolique (ou le mythologique) n'est qu'un aspect (bien que très important) de la représentation de la nature dans les littératures irlandaise et galloise du Moyen Âge. Nous montrerons ainsi que la représentation réaliste de la faune marine, et des poissons en particulier, est liée à l'expérience directe de la nature, partagée par les auteurs des textes examinés et par leur lectorat ${ }^{1}$. Lobjectif final de cet article est de montrer les liens entre les éléments réalistes, mythologiques et religieux dans la représentation de poissons dans les littératures galloise et irlandaise.

\section{LE MONDE RÉEL}

Le saumon, considéré de nos jours comme le poisson le plus emblématique de la mythologie celtique (galloise aussi bien qu'irlandaise), figure dans plusieurs textes du Moyen Âge. Comme remarqué par Boekhoorn, c'est souvent le saumon qui est désigné par référence générique au poisson (Boekhoorn 2008: 261). Selon Joseph Nagy (1986: 127), le saumon fournit un lien entre le monde des hommes et l'au-delà et, en tant qu'intermédiaire, est à son tour particulièrement lié aux poètes et aux savants (voir aussi Boekhoorn 2008: 261). En effet, il est très probable que l'importance du saumon dans la littérature ne soit que la conséquence de sa forte présence dans la réalité quotidienne de la pêche irlandaise.

1. Les traductions des textes citées dans cet article sont de l'auteur, sauf mention contraire. Une partie des textes d'origine galloise cités dans cet article sont aussi disponibles en forme complète en français, avec introduction, dans Fleuriot et al. (1981).
Ce lien entre poésie et pratique représente une tradition qui remonte au Moyen Âge et est encore visible dans le rôle joué par le saumon dans les récits folkloriques des pêcheurs contemporains irlandais (Nagy 1986: 127). Selon les informations fournies par les archéologues, la méthode de pêche la plus fréquente en Irlande consistait en l'utilisation de fish weirs (barrages de pêche/barrages à poisson), technique toujours très répandue dans les périodes plus tardives (Went 1964: 202-207). Ces barrages, dont certains sont encore aujourd'hui visibles, sont les traces les plus importantes de cette activité halieutique irlandaise médiévale. La description fournie par Aidan O'Sullivan (2005) montre que ces structures, construites pour capturer le saumon, la truite de mer, la lamproie, l'anguille et d'autres poissons lors du flux autant que du reflux, pouvaient être de grande ou de petite taille et, pour la plupart, étaient solidement construites. Il apparaît également qu'il existe une permanence de ce type de construction dans les mêmes lieux pendant des siècles (O'Sullivan 2003: 465; 2005: 172). Les mêmes structures de capture de poisson ont été trouvées au Pays de Galles. Bien que ces pièges aient été utilisés pour attraper différentes espèces, le saumon était privilégié et son importance est tout aussi visible dans la poésie irlandaise.

La littérature irlandaise, de même que la galloise, n'est pas particulièrement connue pour ses descriptions réalistes $\mathrm{du}$ saumon. Pourtant, dans un grand nombre d'exemples (notamment en poésie), elle décrit la nature sans aucune référence au surnaturel. La poésie irlandaise sur la nature, et en particulier la poésie associée au nom de Finn Mac Cumaill, nous donne des images exclusivement réalistes (dans le sens d'une absence totale d'éléments mythologiques ou fantastiques) du saumon. En lisant ces poèmes, il devient clair que leurs descriptions du poisson, et plus particulièrement du saumon, reflètent presque toujours la vie réelle observée. Il apparait dans cette poésie, à travers les descriptions non seulement de la vie aquatique mais de la nature en général, que les Irlandais étaient des observateurs perspicaces. De plus, il semble que, plus spécifiquement pour le saumon, les descriptions proviennent de connaissances personnelles, qui sont probablement des résultats de l'expérience directe de la pêche.

Les meilleurs exemples sont fournis par les poèmes sur la nature conservés dans Agallamb na Senórach «La conversation des anciens» (c. 1200), une histoire prosimétrique racontant une conversation entre saint Patrick et Caílte, un membre de la fian, la bande légendaire du Finn (Parsons 2005: 87). Dans plusieurs de ces poèmes, on trouve plusieurs références au poisson, souvent dans un contexte de richesse et de beauté naturelle de la région. Les descriptions sont totalement privées de fantastique et peuvent être analysées comme le reflet d'une bonne connaissance environnementale ${ }^{2}$. Elles présentent un contrepoint très important à l'habituelle image fantastique et surnaturelle du saumon dans la littérature, telle qu'elle est analysée le plus souvent, discussion sur laquelle nous reviendrons plus loin. Une référence au poisson dans ce contexte

2. Comme l'observe Boekhoorn, il y a une association du poisson, et du saumon en particulier, avec les saisons (Boekhoorn 2008: 24). 
de la description de la nature se trouve dans un des poèmes d'Agallamb na Senórach, dans lequel la truite est décrite parallèlement au sanglier:

Do bhric ód bruachaib amach

do mhuca allta it fhásach

(Votre truite sur vos rives

vos cochons sauvages dans vos régions sauvages) (O'Grady 1892: 96).

Selon des études sur l'habitat du poisson, il a été montré que la truite de mer (Salmo trutta Linnaeus, 1758) a tendance à circuler plus près des rives des cours d'eau que le saumon (Salmo salar Linnaeus, 1758), bien que les jeunes saumons, pendant l'année suivant leur naissance, aient aussi tendance à s'approcher plus près des rives que les individus plus âgés (Bremset \& Berg 1999). Il semble donc que le poème différencie ici les deux espèces, saumon et truite, et reflète la réalité du comportement des poissons, telle qu'elle est notamment connue par les pêcheurs. Le mot utilisé pour la truite ici est brecc, emploi substantif du mot qui signifie " tacheté", référant à la couleur de la truite (eDIL s. v. 2 brecc). La même précision se trouve dans un autre poème du même manuscrit, qui fait référence à la truite qui se trouve sous les rives des fleuves de l'île d'Arran: bric fá bruachaib a habann "la truite sous les rives de ses fleuves" (O'Grady 1892: 102). Cela montre qu'au moins pour les poèmes sur la nature, on observe une distinction entre les deux espèces de «saumon " qui, selon Hollard \& Delestrée (2009: 222), sont souvent confondues dans la littérature. Il est possible que cette confusion fréquente soit un phénomène appartenant à la littérature fantastique traitant du saumon, et pas nécessairement à la littérature réaliste, comme dans les poèmes examinés ici. La conclusion la plus importante que l'on puisse déduire de ces poèmes est qu'ils présentent des descriptions réalistes du monde, sans aucun élément mythologique ou fantastique.

Il faut noter que le saumon, de part sa nature itinérante, est un élément ambivalent dans la littérature irlandaise et surtout dans la poésie, où il est parfois associé à la mer ou l'océan, et quelquefois, comme dans les exemples ci-dessus, aux rivières (Boekhoorn 2008: 197, 260, 261). Dans la poésie irlandaise sur la nature, ce poisson est surtout associé aux estuaires. Le premier poème cité ci-dessus contient une autre référence au poisson, plus générale cette fois:

\section{Do mhes ós bharraib do chrann}

tiasc i ninnberaib thabann

(Vos fruits aux sommets de vos arbres

votre poisson dans les estuaires de vos rivières (O'Grady 1892: 96).

Les estuaires sont à la fois les marges visibles entre la mer et la rivière, et les lieux où l'on posait le plus souvent les barrages, tels que ceux décrits plus haut, pour capturer et garder le saumon (O'Sullivan 2003: 451).

Le même reflet d'une expérience directe de la pêche et du poisson dans son habitat naturel apparaît dans la littérature en prose. Dans le texte irlandais Tochmarc Emire « La courtise d'Emer" (Meyer 1890; D'Arbois de Jubainville 1892: 39-50; Van Hamel 1933; Guyonvarc'h 1959), lors d'un dialogue du héros $\mathrm{Cu}$ Chulainn avec Emer, la jeune femme qu'il cour- tise, $\mathrm{Cu}$ Chulainn parle du poisson comme le búar maige Tethrai «bétail de la plaine de Tethra ", c'est-à-dire le poisson (bétail) de la mer (plaine de Tethra) (Van Hamel 1933: 26). Une version courte du récit - plus ancienne - et une version longue - plus tardive - nous sont parvenues (Toner 1998); cette citation provient de la version courte, où la métaphore est déjà transmise. La version longue fournit une explication donnée par Cu Chulainn à Emer. Cette phrase, très illustrative, a des implications pour notre connaissance de la fonction du poisson dans le monde littéraire et culturel de l'Irlande, car elle montre les deux aspects manifestés renvoyant à la vie aquatique dans la littérature irlandaise. Le premier aspect, le plus connu, est l'élément mythique, souvent associé dans la critique moderne aux littératures du monde celtique, et à la littérature irlandaise du Moyen Âge en particulier. Ici, l'aspect mythique est l'utilisation du nom personnel de Tethra dans la phrase "plaine de Tethra», par laquelle $\mathrm{Cu}$ Chulainn veut indiquer la mer. Tethra est un membre d'un peuple connu sous le nom de Fomori. Selon le Lebor Gabála Érenn, le Livre des conquêtes d'Irlande (Macalister 1932, 1933, 1937, 1939, 1942; Carey 2009), il s'agit de l'un des peuples conquérants d'Irlande. L'emploi de son nom donne ici à la phrase un contexte mythologique. Le deuxième aspect de cette phrase est le lien avec le monde réel, évoqué par l'image du poisson comme bétail et la représentation qui en résulte du pêcheur comme berger ${ }^{3}$. $\mathrm{Cu}$ Chulainn évoque une image de poisson dans un enclos, semblable à celui du bétail domestique. On peut se demander si les barrages pour capturer le saumon, construits dans les estuaires sur presque tous les grands cours d'eau d'Irlande, n'avaient pas l'apparence, au moins pour l'auteur de Tochmarc Emire (Meyer 1890), d'un enclos pour le bétail. En tout cas, la métaphore utilisée par $\mathrm{Cu}$ Chulainn montre que le pêcheur était considéré en Irlande comme un équivalent aquatique du berger.

\section{LE SAUMON DU SAVOIR}

\section{Gogwn pet pegor}

yssyd y dan vor;

gogwn eu heissor

pawb yn y oscor (Haycock 2007: 119).

(Je sais combien d'êtres

Il y a sous la mer;

Je sais leur nature

chacun dans son banc).

Ce poème en moyen gallois, intitulé Angar Kyfundawt, titre dont la traduction est difficile mais qui peut être transcrit comme "coalition hostile", est une liste des connaissances du monde naturel, conservée dans une collection de poèmes

3. Dans le Immram Brain cité par Boekhoorn (2008: 264), le saumon est comparé au veau et à l'agneau. Il faut noter que la métaphore utilisée par $\mathrm{Cu}$ Chulainn dans ce texte médiéval n'est pas un élément spécifiquement médiéval et est toujours employée aujourd'hui, notamment dans le nom d'un groupe de protection des écosystèmes marins: Sea Shepherd Conservation Society - bien qu'en ce cas l'emploi du terme shepherd n'ait probablement aucun lien direct avec les textes anciens. 
associés à un personnage appelé Taliesin (Haycock 1997, 2007). Dans la suite du poème, le locuteur prétend savoir pourquoi les poissons sont de couleur noire, et pourquoi ils ont des écailles (Haycock 2007: 120 1. 212, 121 1. 215). Le sujet principal du poème est la connaissance du monde réel, et on pourrait peut-être le classer avec les poèmes réalistes d'Agallamh na Senórach presentés précédemment. Néanmoins ce poème, qui appartient à la légende de Taliesin, est, en partie à cause de son association avec ce personnage, un exemple de la représentation fantastique du monde des poissons.

Dans la littérature galloise, la figure de Taliesin apparaît souvent et est utilisée pour relier le saumon (poisson en lequel Taliesin est transformé selon la légende) avec les concepts de la sagesse et du savoir (Nagy 1986; Marc 2000: 40-49; Walter 2002; Boekhoorn 2008: 260-263). Le saumon est le poisson le mieux étudié de la littérature galloise et irlandaise médiévale, et cette association entre Taliesin et le savoir résulte d'une perception de la représentation de poisson dans les littératures du Pays de Galles et d'Irlande comme étant surtout symbolique et mythologique et en aucun cas de nature réaliste. En effet, dans la littérature galloise, nous n'avons que peu de mentions de poisson, et la plupart de celles-ci concernent le saumon. L'une d'elles se trouve dans l'histoire de Taliesin et l'autre est le saumon de Llyn Llyw dans le récit arthurien le plus ancien du Pays de Galles, Culhwch ac Olwen (c. 1100) (Sterckx 1986: 64; Bromwich \& Evans 1992; Boekhoorn 2008: 190). Selon ce récit, ce poisson est le plus vieil animal du monde, un être savant, et de si grande taille qu'il est capable de porter deux hommes sur ses «épaules» bien que les «épaules» soient difficiles à faire correspondre avec l'anatomie d'un saumon. Ici aussi, nous observons que le saumon est associé à la sagesse.

Revenons à Taliesin: la seconde des deux évocations du saumon dans la littérature galloise du Moyen Âge tient précisément dans l'histoire de sa naissance. Il s'agit ici de la transformation en saumon, attribuée à la figure légendaire de Taliesin dans le récit tardif Ystorya Taliesin (XVe ou XVIe siècle) (Ford $1992)^{4}$. Après avoir bu les trois gouttes magiques du chaudron de Ceridwen, le garçon Gwion Bach essaie d'échapper à sa propriétaire:

«[...] Elle courut à sa poursuite. Quand il l'aperçut, il prit la forme d'un lièvre et se mit à courir. Mais elle se donna la forme d'un lévrier, elle le poursuivit et le chassa vers une rivière. Il prit la forme d'un poisson et elle prit la forme d'une loutre; elle le chercha sous l'eau. Il lui fallut prendre la forme d'un oiseau dans le ciel et elle se fit épervier à sa poursuite. Elle ne lui laissa pas de tranquillité dans le ciel. [...] Il descendit dans le froment et prit la forme d'un grain. Elle prit la forme d'une poule noire à crête [...] le reconnut et l'avala, ainsi que dit l'histoire.» (Guyonvarc'h 1967: 349)

Le résultat de ce processus est la naissance, neuf mois plus tard, de Taliesin, qui est donc une réincarnation de Gwion Bach. Bien que cette légende en prose soit beaucoup plus tardive que les autres textes examinés ici, des traces de cette légende et de l'association de Taliesin avec le saumon datant

4. Dans la littérature galloise du Moyen Âge il y a deux figures de Taliesin personnage historique et personnage légéndaire (Haycock 2006; Koch 2006). d'une période antérieure nous ont été transmises. Dans un poème présumé du XIIe siècle (et possiblement antérieur), préservé dans un célèbre manuscrit du XIVe siècle connu sous le nom de Llyfr Taliesin (Bibliothèque nationale du Pays de Galles, MS Peniarth 2), le narrateur raconte ces transformations magiques. Il mentionne aussi son nom: Taliesin.

Eilgweith y'm rithat:

bum glas gleissat,

bum ki, bum hyd,

bum iwrch ymynyd,

bum kyff, bum raw,

bum bwell yn llaw [...] (Haycock 2007: 121, 122).

(J'ai été de nouveau transformé,

J'ai été un jeune saumon gris,

J'ai été un chien, j'ai été un cerf,

J'ai été un chevreuil dans la montagne,

J'ai été une borne, une corde,

J'ai été une hache dans la main [...]) (Lambert 1993: 334).

Dans ce poème, les références aux poissons ont pour contexte l'association de Taliesin avec le savoir, et particulièrement le savoir encyclopédique du monde de la nature. Cette association parât avoir été bien connue au Moyen Âge, car nous la retrouvons aussi dans la Vita Merlini de Geoffroi de Monmouth (Berthet et al. 1999), dans laquelle Merlin et Taliesin ont une conversation sur le monde du présent (la nature, dont Taliesin est l'expert) et le monde du futur (la prophétie, dont Merlin est l'expert) ${ }^{5}$. Il faut observer que, dans le reste de la légende de Taliesin, ce dernier est particulièrement associé à la prophétie, rôle réservé à Merlin dans la Vita Merlini, alors que les deux devins sont parfois confondus dans la tradition (Walter 2002: 237, 238). Il est aussi possible, bien que difficile à démontrer, que, comme le suggère Philippe Walter (2002: 239, 240), l'association entre Taliesin, le saumon et la prophétie remonte à une période encore plus ancienne.

Un élément important dans le récit de Taliesin fournit un lien entre ce récit fantastique, voire mythologique, et le monde réel dont les lecteurs ou auditeurs de la légende pouvaient avoir l'expérience. Cet élément se trouve dans la partie du récit qui suit la naissance de Taliesin. L'enfant est abandonné dans l'eau et sera plus tard, comme Moïse, trouvé et sauvé. La date de cette découverte de l'enfant dans l'eau varie entre les deux versions survivantes du texte: $1^{\text {er }}$ novembre ou $1^{\text {er }}$ mai. Dans les deux cas, l'association avec la pêche est explicite dans le texte, car les deux dates correspondent en effet à des saisons importantes pour les pêcheurs, celles des migrations du saumon de l'Atlantique (Marc 2000: 43, 44). Ainsi, même le récit fantastique est finalement lié au monde réel, dont le public a une expérience directe.

La transformation en saumon n'est pas spécifique à la littérature galloise, car nous la retrouvons aussi dans un récit prosimétrique irlandais datant du VIIIe ou peut-être même du VIIe siècle, Immram Brain "Voyage de Bran", qui raconte le voyage fantastique et les aventures de Bran Mac Febail (Guyonvarc'h 1957). Dans ce récit, un personnage aux

5. Pour plus de renseignements sur Merlin, voir la bibliographie dans Petrovskaia (2017). 
origines surnaturelles, Manannán, prophétise au sujet d'un autre personnage, Mongán, qu'«il sera un saumon tacheté dans l'eau profonde» (Mac Cana 1972; Sjoestedt 1998: 76; Marc 2000: 48, 49). L'évocation de cette transformation en saumon a moins à voir ici avec le savoir encyclopédique en particulier, mais plus avec l'idée mythique de l'association du saumon avec le pouvoir surnaturel du savoir. Ce n'est plus seulement un personnage qui est transformé en saumon, doté d'une sagesse surnaturelle, mais celui qui parle du saumon et de cette transformation.

Poursuivant les exemples irlandais, un autre héros, Finn Mac Cumaill (personnage à qui la poésie réaliste abordée plus haut est aussi associée), le titulaire d'un cycle littéraire important, est aussi lié avec l'eau et le saumon'. Dans son cas, jeté dans l'eau quand il était petit, il réapparut avec un saumon dans la main (Hemmi 2001: 105). Bien que dans ce récit il n'acquière pas de savoir surnaturel par cette expérience, Finn est dans un autre texte associé à l'idée du savoir, cette fois-ci celui de la poésie. Dans cet autre récit, il lèche son doigt brûlé par des gouttes bouillantes tombant d'un saumon en train de cuire. Le savant à qui était destiné ce saumon issu de l'autre monde et porteur du don de sagesse, reconnait alors qu'il revient à Finn et le lui donne (Meyer 1883, 1904; Walter 2002: 242). Ce conte comporte des similitudes avec le récit gallois de Gwion Bach, qui reçoit aussi le don de sagesse (et du pouvoir magique dans son cas) d'une manière accidentelle, en cuisinant un plat pour un autre destinataire.

\section{L'ÉLÉMENT RELIGIEUX}

Les transformations de Taliesin et l'acquisition du savoir par Finn paraissent être des éléments fantastiques, voire mythologiques, des littératures de Pays de Galles et d'Irlande. Néanmoins, le poisson est aussi utilisé dans des textes à vocation religieuse et chrétienne à la même époque. Dans le récit irlandais Aided Eochaid meic Maireda, une femme, Líban ou Lí Ban, survit à un déluge catastrophique par sa transformation (probablement partielle) en saumon ${ }^{7}$. Elle est finalement trouvée par un saint, transportée dans un récipient en verre, transformée en femme et baptisée (Best \& Bergin 1929: 2958-3132; Nagy 1986: 126, 127). Selon Helen Imhoff (2008), cette histoire doit être interprétée dans un contexte religieux: l'image de "pêcheur des âmes» est représentée littéralement dans le récit. Il reste néanmoins la question intéressante de savoir exactement qui est pêché ici, une femme ou un monstre, et quelle est précisément son apparence physique.

Lí Ban, apparaissant dans le récit comme transformée en être hybride avec une tête de femme et une queue de saumon, se décrit comme un monstre marin : ciapsa duine ciapsa bled [...] (j’étais un être humain ou un monstre marin [...]). Dans un autre manuscrit (Bibliothèque nationale d'Irlande, MS G7), nous avons une autre version de cette description: Ciabsa bled-

6. Pour en savoir plus sur le parallélisme entre les légendes de Finn et de Taliesin, voir Sterckx (1994: 56).

7. Elle apparaît aussi dans des chroniques irlandaises (Imhoff 2008: 113, n. 29).

Pour une discussion sur ce personnage et son nom, voir aussi De Vries (2007). mil nipsa bled (Bien que j'étais une baleine, je n'étais pas un monstre marin). Il est donc difficile de déterminer exactement l'apparence de cette femme-poisson. Sa description d'elle-même nous donne néanmoins un aperçu intéressant de la signification des mots utilisés pour décrire les monstres marins: bled et bledmil: bled en ancien irlandais est utilisé pour baleine et pour monstre marin (eDIL s. v. bled), tandis que bledmilest un mot composé de bled et mil (animal), utilisé pour les monstres marins et baleines, mais aussi pour des reptiles (eDIL s. v. bledmí). Quoi qu'il en soit, ces mots ont une gamme sémantique qui part du réel (la baleine) et va jusqu'aux concepts plus larges de monstre et d'animal marin ${ }^{8}$. Dans ce contexte, il faut aussi observer qu'en tant qu'habitante d'un lac, Lí Ban n'est pas strictement un monstre marin, mais plutôt un être aquatique générique. Son statut est également ambigu, car on ne sait pas si elle a passé toute sa vie dans le lac ou si elle a-aussi vécu dans la mer. Un poème narre qu'elle est destinée à nager tar cach trethain «à travers chaque mer orageuse» (De Vries 2012: 202 $\S 8 \mathrm{~b}$ ), le mot trethain signifiant «mer», et particulièrement la mer tempétueuse (eDIL s. v. 1 trethan). La même ambiguïté de signification peut être observée pour l'autre mot utilisé dans le contexte des voyages de Lí Ban: muir (la mer) (De Vries 2012: $202 \$ 9$; eDIL s. v. muir). Cette ambiguité dans la figure de Lí Ban, associée à l'eau douce aussi bien qu'à l'eau salée, est probablement le résultat de sa connexion avec le saumon, poisson itinérant. Un autre parallèle peut être fait car elle est accompagnée par son chien, lui aussi transformé en loutre. La loutre dans la littérature irlandaise est souvent proche du saumon, comme un compagnon de ce poisson, mais il est possible qu'il s'agisse d'un jeu de mot, car en irlandais le mot désignant la loutre est doborchú, c'est-à-dire le "chien d'eau» (Nagy 1986; De Vries 2012: 167 n.466; eDIL s. v. onchî) ${ }^{9}$.

\section{LE RÔLE DE L'EXPÉRIENCE DU RÉEL DANS LA LITTÉRATURE FANTASTIQUE OU RELIGIEUSE}

La poursuite des monstres marins dans la littérature religieuse de l'Irlande devait nous amener tôt ou tard à la Navigatio sancti Brendani abbatis, texte latin composé probablement en Irlande, bien que connu principalement par des manuscrits continentaux (Strijbosch 2006: 1, 2, 6; Zelzer 2006: 337, 338; Mackley 2008: 1, 14, 15), et dont il existe plusieurs versions (Burgess \& Strijbosch 2000). Un contraste intéressant dans la représentation du monde marin existe entre le texte latin de la Navigatio et sa traduction anglo-normande réalisée par Benedeît (XIIe siècle), le Voyage de Saint Brandan (Selmer 1959) ${ }^{10}$. Le monstre aquatique le plus fameux dans I'histoire de Brendan est «Jasconius»,

8. Ce personnage, et son association avec le saumon, est mentionné par Boekhoorn (2008: 264, 267), qui cependant n'examine pas son association potentielle avec la baleine.

9. Il faut observer qu'une construction parallèle est attestée dans le gallois (dyfrgi) et le breton (dourgi) (Le Bihan pers. com.). Voir GPC s.v. dyfrgi, dwrgi http://geiriadur.ac.uk/gpc/gpc.html dernière consultation: 02/07/2018.

10. Pour un aperçu des autres versions vernaculaires de la Navigatio, voir Selmer (1956); pour une bibliographie des études de la légende de Brendan, voir Burgess \& Strijbosch (2000). Pour plus sur l'identité de Benedeît, voir Mackley (2008: 32, 33). 
représenté comme un poisson-île. Présent dans les différentes versions de la légende de Brendan, cet animal, identifié comme une baleine, a été l'objet d'un grand nombre d'études dont un aperçu complet n'est pas possible ici (Bieler 1947; Hernández González 1993; Borsje 1996: 124-128; Burgess 2006: 14; Iannello 2013). Jasconius ayant été bien étudié, nous nous concentrerons sur les autres monstres marins qui apparaissent dans ce texte. C'est précisément dans les descriptions de ces monstres, et notamment dans la première bataille entre ceuxci, que la différence est la plus grande entre la version latine et la version anglo-normande de Benedeît. La scène décrit une bataille entre deux monstres marins, dont le premier fait peur aux moines quand il apparaît, parce qu'il semble vouloir avaler Brendan, ses compagnons et leur bateau. Dans le Voyage, cette bête est définitivement fantastique:

\section{Li fus de lui si enbraise}

Cume buche de fornaise:

La flamme est grant, escalfed fort [...] (Short \& Merrilees 1984: 905-909).

Au contraire, dans le texte latin, cette créature marine - probablement une baleine - ne possède aucune caractéristique fantastique. Bien que géante, elle ne fait que produire de l'écume de ses narines (Selmer 1959; Borsje 1996: 128). Ce contraste entre les descriptions des monstres trouve un parallèle dans celle de la bataille car, comme l'observe Glyn Burgess (2006: 19), la description est beaucoup plus développée dans la version de Benedeît.

Il nous reste à essayer d'expliquer cette différence entre la version de Benedeît, avec ses éléments fantastiques, et le texte latin totalement dépourvu de tels éléments. Il a déjà été observé par Strijbosch que Benedeît a adapté le texte selon les attentes de son public (Strijbosch 2006: 3, 7). Nous suggérons que la différence entre le texte irlandais « réaliste» et son adaptation fantastique anglo-normande est le résultat direct des différences entre les deux lectorats destinataires de ces deux textes. Mackley identifie trois types de public pour la Navigatio: les frères du monastère, les pèlerins que le monastère voulait attirer, et la communauté entourant les monastères (Mackley 2008: 17-19). C'est ce troisième type de public, la communauté élargie, qui formait selon Mackley le lectorat principal envisagé par l'auteur de la Navigatio (Mackley 2008: 19) et c'est pour lui, semblet-il, que les éléments réalistes ont été inclus dans la version latine. Ce groupe comprenait probablement des pêcheurs qui avaient une connaissance directe de la mer et de ses habitants. Comme l'observe Michela Zelzer (2006: 341), il est bien possible qu'en arrière-plan du récit de la navigation de Brendan se trouvent des expériences réelles des Irlandais sur l'océan. La forme du récit et son but sont certainement liés à la liturgie et la vie monastique, mais les détails de la mer ne sont pas nécessairement uniquement des symboles. En effet, comme le signale Jude Mackley (2008: 2, 3), les éléments réalistes (comme, par exemple, des îles qui peuvent être identifiées) ne diminuent pas l'importance du fantastique dans ce texte. À propos de la topographie, Mackley fait une observation qui est aussi valable pour les descriptions du monde aquatique: l'auteur de la Navigatio donne à son public des éléments qu'il connaissait déjà (Mackley 2008: 15, 16).
En revanche, comme l'observe Mackley (2008: 31), la version de Benedeît fait le choix d'omettre des éléments qui n'auraient pas d'intérêt pour son public anglo-normand, tels que la généalogie de Brendan et la topographie de l'Irlande. Glynn Burgess, qui a étudié l'utilisation des animaux (y compris les poissons et les monstres marins) dans le texte de Benedeît, a observé que le traducteur utilise les animaux dans le récit pour réconcilier les éléments liturgiques (et monastiques) avec les habitudes littéraires de son public - un public courtois (Burgess 2006: 33, 34). Il faut ajouter que l'élément de description réaliste des jets d'eau émis par la baleine aurait été sans doute trop banal pour le public courtois et aurait ainsi été remplacé dans la traduction par le feu. Il est probable que pour le public aristocratique du Voyage (Mackley 2008: 38), qui n'avait pas l'expérience directe de la mer, la description qui figurait dans le texte latin n'aurait pas été assez intéressante. Cependant, dans la Navigatio latine, c'est le deuxième monstre, qui arrive pour combattre le premier et ainsi sauver les moines, qui produit des flammes. Cet élément fantastique peut paraître contradictoire dans le contexte de l'hypothèse proposée ici, mais il faut se souvenir que ce deuxième monstre n'apparaît que lorsque Brendan et les moines prient Dieu pour les sauver du premier. On peut ainsi postuler que, tandis que le premier monstre marin est un être naturel (connu par l'expérience directe ou indirecte du public de ce texte), le deuxième est le résultat direct de l'intervention divine et peut ainsi, et même doit, avoir des caractéristiques fantastiques. La distinction n'est plus nécessaire dans la version anglo-normande, car le lectorat n'a pas l'expérience de la mer et peut tranquillement imaginer que des êtres qui l'habitent peuvent avoir toutes sortes de caractéristiques monstrueuses. Le monde du fantastique commence pour eux à partir de ce moment, et pas comme le résultat direct de l'intervention divine.

Un argument très proche a été présenté par Mackley, qui suggère que le monstre dans la Navigatio est, bien que merveilleux, contextualisé par la présence précédente de Jasconius dans le texte, et de ce fait représente quelque chose de déjà connu et déjà compris par le public (Mackley 2008: 160). Notre suggestion est que ce n'est pas seulement Jasconius, mais aussi les connaissances préalables du lecteur de la Navigatio, qui fournissent le contexte à cette bête (et qui limitent la description à ce qui est strictement possible). En revanche, dans le Voyage, le merveilleux n'a pas de restrictions semblables.

\section{CONCLUSIONS}

Nous pouvons établir quelques conclusions à partir du matériel littéraire examiné dans le présent article. Pour commencer, il est clair qu'il existait un élément réaliste très important dans les descriptions de la nature dans les œuvres irlandaises les plus anciennes, telles que la poésie associée à Finn Mac Cumaill, conservée dans Agallamh na Senórach (Parsons 2005). Cette poésie réaliste reflète l'expérience de la nature et de la pêche, en particulier au Moyen Âge, tandis que dans d'autres textes, tels que Tochmarc Emire (Meyer 1890), nous retrouvons des 
images de poisson comme bétail, qui rappellent les barrages de pêche utilisés à cette période. Finn Mac Cumaill, associé à la poésie réaliste, est aussi lié à l'image fantastique, voire mythique, du saumon de savoir. Cette association du don poétique et prophétique (car poésie et prophétie vont de pair dans les littératures d'Irlande et du Pays de Galles au Moyen Âge) est aussi attestée dans la littérature du Pays de Galles, en la figure légendaire de Taliesin. Bien que cette association entre saumon et don du savoir dans les deux traditions ait été l'aspect le plus étudié de la représentation du poisson dans les littératures celtiques, cet article a montré que ce n'était qu'un moyen de représenter le monde aquatique.

C'est dans les textes qui portent des significations religieuses chrétiennes, tels que Aided Eochaid meic Maireda (Best \& Bergin 1929) et la Navigatio sancti Brendani (Selmer 1959), que l'on trouve l'influence de l'observation du monde réel. La femme transformée en saumon vit dans un lac, mais sa nature de saumon dicte qu'elle doit être identifiée à un être marin. L'illustration la plus significative est fournie par le contraste entre le texte latin de la Navigatio, composé en Irlande pour un public irlandais qui connaissait bien la mer, et le texte anglo-normand du Voyage de Saint Brendan, composé pour un public courtois éloigné de l'expérience directe de la pêche et des animaux marins. Dans le texte latin, le monstre rencontré naturellement est une baleine, représentée sans éléments fantastiques, telle qu'on peut la trouver en plein océan; c'est le second monstre, qui apparaît comme une réponse divine, qui porte des caractéristiques fantastiques. Dans le texte anglo-normand, il n'y a pas de raison pour rester fidèle à ce qui est possible, car le public ne peut utiliser son expérience personnelle pour contredire l'auteur. Il apparaît donc que les éléments fantastiques de la description augmentent proportionnellement au manque de connaissance directe de la faune marine des lecteurs ou auditeurs de l'œuvre. Nous pouvons ainsi conclure que la fidélité au réel était plus importante pour les auteurs des textes irlandais et gallois du Moyen Âge qu'on ne l'a cru initialement.

\section{RÉFÉRENCES}

BEST R. I. \& BERGIN O. (eds) 1929. - Lebor na hUidre. Book of the Dun Cow. Royal Irish Academy, Dublin, xliv + 341 p.

Bieler L. 1947. - Casconius, the monster of the Navigatio Brendani. Égse, a Journal of Irish Studies (5): 139, 140.

BOEKHOORN D. N. 2008. - Bestiaire mythique, légendaire et merveilleux dans la tradition celtique: de la littérature orale à la littérature écrite: étude comparée de l'évolution du rôle et de la fonction des animaux dans les traditions écrites et orales ayant trait à la mythologie en Irlande, Écosse, Pays de Galles, Cornouailles et Bretagne à partir du Haut Moyen Âge, appuyée sur les sources écrites, iconographiques et toreutiques chez les Celtes anciens continentaux. Thèse de doctorat en littérature. Université Rennes 2, Rennes; University College Cork, Cork, 451 p. https://tel.archives-ouvertes.fr/tel-00293874/ document dernière consultation: 02/07/2018.

Berthet J.-C., Bord C. \& Stalmans N. 1999. - Vie de Merlin par Geoffrey de Monmouth, in WALTER P. (éd.), Le divin maudit: Merlin, Lailoken, Suibhne. Textes et études. ELLUG, Grenoble: 49-171. (Coll. Moyen Âge européen).

BORSJE J. 1996. - From Chaos to Enemy: Encounters with Monsters in Early Irish Texts. An Investigation Related to the Process of Chris- tianization and the Concept of Evil. Brepols, Turnhout, 430 p. (Coll. Instrumenta Patristica; 29).

Bremset G. \& Berg O. K. 1999. - Three-dimensional microhabitat use by young pool-dwelling Atlantic salmon and brown trout. Animal Behaviour 58 (5): 1047-1059 https://doi.org/10.1006/ anbe.1999.1218

Bromwich R. \& Evans D. S. (eds) 1992. - Culhwch ac Olwen: An Edition and Study of the Oldest Arthurian Tale. University of Wales Press, Cardiff, lxxxiii +226 p.

BURGESS G. S. 2006. - The use of animals in Benedeit's version of the Brendan legend, in Burgess G. S. \& STRIJBOSCH C. (eds), The Brendan Legend: Texts and Versions. Brill, Leiden: 11-34.

Burgess G. S. \& STRIjBosch C. (eds) 2000. - The Brendan Legend. A Critical Bibliography. Royal Irish Academy, Dublin, ix $+297 \mathrm{p}$

CAREY J. (ed.) 2009. - Lebor Gabála Érenn: Textual History and Pseudohistory. Irish Texts Society, Dublin, $\mathrm{x}+114 \mathrm{p}$. (Coll. Irish Texts Society. Subsidiary Series; 20).

D’ARbois De JubainVILle H. (trad.) 1892. - Cours de littérature celtique 5: L'épopée celtique en Irlande. Tome 1er. Thorin, Paris, xliv $+533 \mathrm{p}$.

De VRIES R. 2007. - The names of Li Ban, in NAGY J. F. (ed.), Myth in Celtic Literatures. Four Courts, Dublin: 39-54. (Coll. CSANA Yearbook; 6).

De VRIES R. 2012. — Two texts on Loch nEchach: De causi storchi Corc' Óche and Aided Echachmaic Maireda. Irish Texts Society Main Series 65: xii + 328 p.

Fleuriot L., Lozac'Hmeur J.-C. \& Prat L. C. 1981. — Récits et poèmes celtiques: domaine brittonique VIe-XVe siècles. Stock, Paris, 250 p.

Ford P. K. (ed.) 1992. - Ystoria Taliesin. University of Wales Press, Cardiff, xvi $+175 \mathrm{p}$

GuYONVARC'H C.-J. (trad.) 1957. — La navigation de Bran fils de Febal. Ogam (9): 304-309.

GuYONVARC'H C.-J. (trad.) 1959. - La courtise d'Émer. Ogam (11): 413-423

GuYONVARC'H, C.-J. (trad.) 1967. — L'histoire de Taliesin. Ogam (19): 348-350.

HAYCOCK M. 1997. - Taliesin's questions. Cambrian Medieval Celtic Studies (33): 19-80.

HAYCOCK M. 2006. - Taliesin. 2: the Taliesin tradition, in KoCH J. T. (ed.), Celtic Culture: a Historical Encyclopedia. ABC Clio, Santa Barbara, Denver, Oxford: 1653-1656.

HaYCOCK M. (ed. \& trad.) 2007. — Legendary Poems from the Book of Taliesin. Cambrian Medieval Celtic Studies Press, Aberystwyth, viii +559 p.

Hemmi Y. 2001. - Celtic Heroes, 'Changelings', and the Mothers. 慶應義塾大学日吉紀要. 英語英米文学 [Keio gijyukudaigakuhiyoshikiyou. eigoeibungaku] 37 (3): 93-116 http://koara.lib.keio.ac.jp/ xoonips/modules/xoonips/image.php/file/10066/AN1003006020000930-0093 dernière consultation : 02/07/2018.

Hernández GonzÁlez F. 1993. - El episodio de la ballena en la Navigatio Sancti Brendani y su precedente en el Physiologus. Fortunatae 5: 283-307.

Hollard D. \& Delestrée L.-P. 2009. — Numismatique gauloise et mythozoologie celtique: à propos de monnaies inédites "au saumon" des Aulerci Eburovices et des Veliocassi, in VAN HEESCH J. \& HeEren I. (eds), Coinage in the Iron Age: Essays in Honour of Simone Scheers. Spink, London: 221-229.

IANNELLO F. 2013. - Jasconius Rivelato. Studio comparative del simbolismo religioso dell'"isola balena" nella Navigatio Sancti Brendani. Edizioni dell'Orso, Alessandria, xxi + 638 p. (Coll. Biblioteca di studi storico-religiosi; 9).

IMHOFF H. 2008. - The themes and structure of Aided Echach maic Maireda. Ériu 58: 107-131. https://doi.org/10.3318/ ERIU.2008.58.107

KocH J. T. 2006. - Taliesin. 1: the historical Taliesin, in $\mathrm{KocH}$ J. T. (ed.), Celtic Culture, a Historical Encyclopedia. ABC Clio, Santa Barbara, Denver, Oxford: 1652, 1653. 
LAMBERT P.-Y. (trad.) 1993. - Les quatre branches du Mabinogi et autres contes gallois du Moyen Agge. Gallimard, Paris, 419 p. (Coll. L'aube des peuples).

MACAlister R. A. S. (ed.) 1932. — Lebor Gabála Érenn: the Book of the Taking of Ireland Vol. 1. Irish Texts Society, Dublin, $352 \mathrm{p}$. (Coll. Irish Texts Society; 34).

MaCalister R. A. S. (ed.) 1933. Lebor Gabála Érenn: the Book of the Taking of Ireland. Vol. 2. Irish Texts Society, Dublin, 273 p. (Coll. Irish Texts Society; 35).

MaCalisTer R. A. S. (ed.) 1937. — Lebor Gabála Erenn: the Book of the Taking of Ireland. Vol. 3. Irish Texts Society, Dublin, $230 \mathrm{p}$. (Coll. Irish Texts Society; 39).

MACALISTER R. A. S. (ed.) 1939. — Lebor Gabála Érenn: the Book of the Taking of Ireland. Vol. 4. Irish Texts Society, Dublin, $342 \mathrm{p}$. (Coll. Irish Texts Society; 41).

MACALISTER R. A. S. (ed.) 1942. - Lebor Gabála Érenn: the Book of the Taking of Ireland. Vol. 5. Irish Texts Society, Dublin, 606 p. (Coll. Irish Texts Society; 44).

MAC CANA P. 1972. - Mongán Mac Fiachna and Immram Brain. Ériu 23: 102-142.

MaCKLEY J. S. 2008. - The Legend of St Brendan: a Comparative Study of the Latin and Anglo-Norman Versions. Brill, Leiden, $\mathrm{x}+$ 350 p. (Coll. The Northern World; 39). https://doi.org/10.1163/ ej.9789004166622.i-352

MARC C. 2000. - Le Fils du Roi des Poissons. Étude Comparative du conte AT 303 et de récits médiévaux. Thèse de doctorat. Université de Grenoble, Grenoble, 770 p.

MeYER K. (ed.) 1883. - Macgnimartha Find. Revue celtique 5: 195-204.

MEYER K. (ed. \& trad.) 1890. — The oldest version of Tochmarc Emire. Revue celtique 11: 433-457.

MeYer K. (trad.) 1904. — The Boyish exploits of Finn. Ériu (1): 180-190.

MEYER K. (ed. \& trad.) 1919. - Bruchstücke der Älteren Lyrik Irlands. Verlag der Akademie der Wissenschaften, Berlin, $72 \mathrm{p}$. http://sulis.ucc.ie/cdi/wp-content/uploads/textarchive/Bruckstucke_der_alteren_Lyrik_Irlands_KMeyer0001.pdf dernière consultation: 02/07/2018.

NAGY J. F. 1986. - Otter, salmon, and eel in traditional Gaelic narrative. Studia Celtica (20-21): 123-144.

O'Grady S. H. (ed. \& trad.) 1892. - Silva Gadellica (I-XXXI): a Collection of Tales in Irish with Extracts Illustrating Persons and Places. Vol. 1: Irish Text. Williams \& Norgate, London, 634 p.

O'SUlLIVAN A. 2003. - Place, memory and identity among estuarine fishing communities: interpreting the archaeology of early medieval fish weirs. World Archaeology 35: 449-468. https://doi. org/10.1080/0043824042000185810

O'Sullivan A. 2005. - Fishing, in Duffy S. (ed.), Medieval Ireland: an Encyclopedia. Routledge, New York, London: 289-292.
PArsons G. 2005. - Acallam Na Sénorach as Prosimetrum. Proceedings of the Harvard Celtic Colloquium 24-25: 86-100 www. jstor.org/stable/40285183

PeTROVSKAIA N. I. 2017. — The fool and the wise man: the legacy of the two Merlins in modern culture, in NELSON-CAMPBELL D. \& CHOLAKIAN R. (eds), The Legacy of Courtly Literature: from Medieval to Contemporary Culture. Palgrave Macmillan, Cham: 173-203. https://doi.org/10.1007/978-3-319-60729-0_10

SELMER C. 1956. - The vernacular translations of the Navigatio Sancti Brendani: a bibliographical study. Mediaeval Studies 18: 145-157. https://doi.org/10.1484/J.MS.2.306607

Selmer C. (ed.) 1959. - Navigatio Sancti Brendani Abbatis from Early Latin Manuscripts. University of Notre Dame Press, Notre Dame IN, li + 132 p. (Coll. Publications in Medieval Studies; 16).

Short I. \& Merrilees B. S. (éds) 1984. - Le voyage de SaintBrandan par Benedeit. Union générale d'éditions, Paris, 142 p. (Coll. 10-18. Série Bibliothèque médiévale).

Sjoestedt M.-L. 1998. — Dieux et héros des celtes. Terre de brume, Rennes, $158 \mathrm{p}$.

STERCKX C. 1986. - Eléments de cosmogonie celtique. Université de Bruxelles, Bruxelles, $127 \mathrm{p}$.

STERCKX C. 1994. - Les dieux protéens des celtes et des indo-européens. Ollodagos, Bruxelles, 201 p. (Coll. Mémoires de la Société belge d'Études celtiques; 4).

STERCKX C. 1996. - Dieux d'eau: Apollons celtes et gaulois. Université de Bruxelles, Bruxelles, 186 p. (Coll. Mémoires de la Société belge d'Études celtiques; 6 ).

STRIJBOSCH C. 2006. - Searching for a versatile saint: introduction, in Burgess G. S. \& STRIjBOSCH C. (eds), The Brendan Legend: Texts and Versions. Brill, Leiden: 1-9. (Coll. The Northern World; 24).

TONER G. 1998. - The Transmission of "Tochmarc Emire". Ériu 49: 71-88. https://www.jstor.org/stable/30007845

Van Hamel A. G. (ed.) 1933. - Compert Con Culainn and Other Stories. Dublin Institute for Advanced Studies, Dublin, xii + 223 p. (Coll. Mediaeval and Modern Irish; 3 ).

WaLTER P. 2002. - Taliesin, homme-saumon, in JAMEs-RAOUL D. \& THOMASSET C. A. (éds), Dans l'eau, sousl'eau: le monde aquatique au Moyen Âge. Presses de l'Université de Paris-Sorbonne, Paris: 237-251.

Went A. E. J. 1964. - The pursuit of salmon in Ireland. Proceedings of the Royal Irish Academy: Archaeology, Culture, History, Literature 63: 191-244. https://www.jstor.org/stable/25505116

ZelZer M. 2006. — Philological remarks on the so-called Navigatio s. Brendani, in STRIJBOSCH C. \& Burgess G. S. (eds), The Brendan Legend: Texts and Versions. Brill, Leiden: 337-350.

\section{SITE INTERNET}

EDIL, electronic Dictionary of the Irish Language: http://dil.ie/ dernière consultation: 02/07/2018. 